\title{
GENERALIZED POWER DOMINATION IN REGULAR GRAPHS*
}

\author{
PAUL DORBEC ${ }^{\dagger}$, MICHAEL A. HENNING ${ }^{\ddagger}$, CHRISTIAN LÖWENSTEIN $^{\ddagger}$, MICKAEL \\ MONTASSIER $^{\dagger}$, AND ANDRÉ RASPAUD ${ }^{\dagger}$
}

\begin{abstract}
In this paper, we continue the study of power domination in graphs (see [T. W. Haynes et al., SIAM J. Discrete Math., 15 (2002), pp. 519-529; P. Dorbec et al., SIAM J. Discrete Math., 22 (2008), pp. 554-567; A. Aazami et al., SIAM J. Discrete Math., 23 (2009), pp. 1382-1399]). Power domination in graphs was birthed from the problem of monitoring an electric power system by placing as few measurement devices in the system as possible. A set of vertices is defined to be a power dominating set of a graph if every vertex and every edge in the system is monitored by the set following a set of rules (according to Kirschoff laws) for power system monitoring. The minimum cardinality of a power dominating set of a graph is its power domination number. We show that the power domination of a connected cubic graph on $n$ vertices different from $K_{3,3}$ is at most $n / 4$ and this bound is tight. More generally, we show that for $k \geq 1$, the $k$-power domination number of a connected $(k+2)$-regular graph on $n$ vertices different from $K_{k+2, k+2}$ is at most $n /(k+3)$, where the 1 -power domination number is the ordinary power domination number. We show that these bounds are tight.
\end{abstract}

Key words. power domination, electrical network monitoring, domination, regular graphs

AMS subject classification. 05C69

DOI. $10.1137 / 120891356$

1. Introduction. In this paper we continue the study of the power domination in graphs started in $[3,13]$ and which is now well-studied in the literature (see, for example, $[1,2,3,5,6,7,8,9,11,12,13,14,15,16])$.

For notation and graph theory terminology not defined herein, we in general follow [10]. In this paper we only consider simple graphs, which are graphs without multiple edges or loops. Let $G=(V, E)$ be a graph with vertex set $V=V(G)$, edge set $E=E(G)$, order $n(G)=|V|$, and size $m(G)=|E|$. The open neighborhood of a vertex $v \in V$ is $N_{G}(v)=\{u \in V \mid u v \in E\}$, and the degree of $v$ is $d_{G}(v)=\left|N_{G}(v)\right|$. The closed neighborhood of $v$ is the set $N_{G}[v]=N_{G}(v) \cup\{v\}$. The open neighborhood of a subset $S \subseteq V$ of vertices is the set $N_{G}(S)=\cup_{v \in S} N(v)$, while the closed neighborhood of $S$ is the set $N_{G}[S]=N_{G}(S) \cup S$. The open neighborhood of $v$ in the set $S$ is the set $N_{S}(v)=N_{G}(v) \cap S$, while the closed neighborhood of $v$ in the set $S$ is the set $N_{S}[v]=N_{G}[v] \cap S$. If the graph $G$ is clear from the context, we simply write $n, m$, $N(v), N[v], N(S), N[S]$, and $d(v)$ rather than $n(G), m(G), N_{G}(v), N_{G}[v], N_{G}(S)$, $N_{G}[S]$, and $d_{G}(v)$, respectively.

The graph $G$ is a $k$-regular if $d(v)=k$ for every vertex $v \in V$. A regular graph is a graph that if $k$-regular for some $k \geq 0$. The complete bipartite graph with partite sets of cardinality $i$ and $j$ we denote by $K_{i, j}$. The graph obtained from $K_{i, j}$ by deleting one edge we denote by $K_{i, j}-e$.

*Received by the editors September 13, 2012; accepted for publication (in revised form) July 8, 2013; published electronically September 19, 2013.

http://www.siam.org/journals/sidma/27-3/89135.html

†University Bordeaux, LaBRI, UMR 5800, F-33400 Talence, France and CNRS, LaBRI, UMR 5800, F-33400 Talence, France (dorbec@labri.fr, montassi@labri.fr, raspaud@labri.fr). The research of these authors was supported in part by ANR/NSC contract Gratel: ANR09-blan-0373-01.

${ }^{\ddagger}$ Department of Mathematics, University of Johannesburg, Auckland Park 2006, South Africa (mahenning@uj.ac.za, christian.loewenstein@uni-ulm.de). The second author's research was supported in part by the South African National Research Foundation and the University of Johannesburg. 
For a set $S \subseteq V$, we let $G[S]$ denote the subgraph induced by $S$. The graph obtained from $G$ by deleting the vertices in $S$ and all edges incident with vertices in $S$ is denoted by $G-S$. In the special case when $S=\{v\}$, we also denote $G-S$ by $G-v$ for simplicity. If $w \in V$, then the degree of $w$ in $S$, denoted $d_{S}(w)$, is the number of vertices in $S$ adjacent to $w$, that is, $d_{S}(w)=|N(w) \cap S|$. In particular, $d_{V}(w)=d(w)$. The set $S$ is an independent set (also called a stable set in the literature) if no two vertices of $S$ are adjacent in $G$, while the set $S$ is a packing if the vertices in $S$ are pairwise at distance at least 3 apart in $G$.

For subsets $X, Y \subseteq V$, we denote the set of edges that join a vertex of $X$ and a vertex of $Y$ by $[X, Y]$. Thus, $|[X, Y]|$ is the number of edges with one end in $X$ and the other end in $Y$. In particular, $|[X, X]|=m(G[X])$. If all possible edges in $[X, Y]$ are present, we say that $[X, Y]$ is full. If there is no edge in $[X, Y]$, we say that $[X, Y]$ is empty. If $X, Y \subseteq V$, then the set $X$ is said to dominate the set $Y$ if $Y \subseteq N[X]$. In particular, if $X$ dominates $V$, then $N[X]=V$ and the set $X$ is called a dominating set in $G$. Thus if $X$ is a dominating set in $G$, then every vertex $v \in V$ is either in $X$ or adjacent to a vertex of $X$. The domination number of $G$, denoted by $\gamma(G)$, is the minimum cardinality of a dominating set.

The notion of power domination in graphs was introduced in $[3,13]$ to model the problem of monitoring electrical networks and was first described as a graph theoretical problem in [9]. The problem has a domination flavor to it, but in addition to domination properties there is the possibility of some propagation according to Kirschoff laws. The original definition of power domination, which required the system to monitor both edges and vertices, was simplified to the following definition independently in $[5,6]$.

Let $G=(V, E)$ be a graph and let $S \subseteq V$ be a subset of its vertices. The set monitored by $S$, denoted by $M(S)$, is defined algorithmically as follows:

- (domination) $M(S) \leftarrow S \cup N(S)$,

- (propagation) as long as there exists $v \in M(S)$ such that $N(v) \cap(V(G)-$ $M(S))=\{w\}$, set $M(S) \leftarrow M(S) \cup\{w\}$.

Equivalently, the set $M(S)$ of vertices monitored by the set $S$ is obtained from $S$ as follows. Initially, the set $M(S)$ consists of the vertices from the closed neighborhood of $S$, that is, $M(S)$ consists of all vertices dominated by $S$. Thereafter we repeatedly add to $M(S)$ vertices $w$ that have a neighbor $v$ in $M(S)$ such that all the other neighbors of $v$ are already in $M(S)$. We continue this process until no such vertex $w$ exists, at which stage the set monitored by $S$ has been constructed. The set $S$ is called a power dominating set of $G$, abbreviated PD-set, if $M(S)=V$ and the power domination number $\gamma_{\mathrm{P}}(G)$ is the minimum cardinality of a PD-set in $G$.

As remarked in [4], the definition of a power dominating set implies some propagating behavior of the set of monitored vertices, a phenomenon very different from the standard domination parameter. Power domination is now well-studied in graph theory. From the algorithmic and complexity point of view, the power domination problem is known to be NP-complete $[1,2,7,8,9]$, and approximation algorithms were given, for example, in [2]. On the other hand, linear-time algorithms for the power domination problem were given for trees [9], for interval graphs [12], and for block graphs [14]. Parameterized results were given in [11]. The exact values for the power domination numbers are determined for various products of graphs in $[5,6]$. Bounds for the power domination numbers of connected graphs and of claw-free cubic graphs are given in [16], and for planar or outerplanar graphs with bounded diameter in [15].

Motivated by Aazami's [1] work on domination in graphs with bounded propagation, Chang et al. [4] defined generalized power domination in graphs. For $k \geq 0$ an 
integer, they introduce the concept of $k$-power domination in graphs as a natural generalization of power domination, with correspondence when $k=1$. When $k=0$, their definition also generalizes usual domination, thereby unifying the seemingly unrelated notions of power domination and ordinary domination in graphs.

Definition 1.1 (monitored set). Let $G=(V, E)$ be a graph and let $S \subseteq V$. For $k \geq 0$, we define the sets $\left(\mathcal{P}_{G}^{i}(S)\right)_{i \geq 0}$ of vertices monitored by $S$ at step $i$ by the following rules:

- $\mathcal{P}_{G}^{0}(S)=N[S]$.

- $\mathcal{P}_{G}^{i+1}(S)=\bigcup\left\{N[v]: v \in \mathcal{P}_{G}^{i}(S)\right.$ such that $\left.\left|N[v] \backslash \mathcal{P}_{G}^{i}(S)\right| \leq k\right\}$.

We remark that for $i \geq 0$ we have $\mathcal{P}_{G}^{i}(S) \subseteq \mathcal{P}_{G}^{i+1}(S) \subseteq V$. Furthermore if a vertex $v$ in the set $\mathcal{P}_{G}^{i}(S)$ has at most $k$ neighbors outside the set, then the set $\mathcal{P}_{G}^{i+1}(S)$ contains $N[v]$. As observed in [4], if $\mathcal{P}_{G}^{i_{0}}(S)=\mathcal{P}_{G}^{i_{0}+1}(S)$ for some $i_{0}$, then $\mathcal{P}_{G}^{j}(S)=\mathcal{P}_{G}^{i_{0}}(S)$ for every $j \geq i_{0}$ and we accordingly define $\mathcal{P}_{G}^{\infty}(S)=\mathcal{P}_{G}^{i_{0}}(S)$. If the graph $G$ is clear from the context, we simply write $\mathcal{P}^{i}(S)$ and $\mathcal{P}^{\infty}(S)$ rather than $\mathcal{P}_{G}^{i}(S)$ and $\mathcal{P}_{G}^{\infty}(S)$. We are now in a position to state the definition of a $k$-power dominating set in a graph first defined by Chang et al. [4].

Definition 1.2 ( $k$-power dominating set). Let $G=(V, E)$ be a graph, let $S \subseteq V$, and let $k \geq 0$ be an integer. If $\mathcal{P}_{G}^{\infty}(S)=V$, then the set $S$ is called a $k$-power dominating set of $G$, abbreviated $k P D$-set. The minimum cardinality of a $k P D$-set in $G$ is called the k-power domination number of $G$, written $\gamma_{\mathrm{P}, k}(G) . A \gamma_{\mathrm{P}, k}(G)$-set is a kPD-set in $G$ of cardinality $\gamma_{\mathrm{P}, k}(G)$.

2. Main result. Our aim in this paper is to establish a sharp upper bound on the $k$-power domination number of a connected $(k+2)$-regular graph in terms of its order for all $k \geq 1$. Corresponding to the case when $k=1$, Zhao, Kang, and Chang proved in [16] that the power domination number of connected claw-free cubic graphs is at most $\frac{n}{4}$. Later, Chang et al. [4] generalized this result and proved that connected claw-free $(k+2)$-regular graphs of order $n$ have a $k$-power domination number at most $\frac{n}{k+3}$. In this paper, we prove that the claw-free condition can be dropped, and thus prove the following result.

THEOREM 2.1. Let $k \geq 1$ and let $G$ be a connected $(k+2)$-regular graph of order $n$. If $G \neq K_{k+2, k+2}$, then $\gamma_{\mathrm{P}, k}(G) \leq n /(k+3)$, and this bound is tight.

We remark that as a special case of Theorem 2.1 when $k=1$, we have that if $G \neq K_{3,3}$ is a connected cubic regular graph of order $n$, then $\gamma_{\mathrm{P}}(G) \leq n / 4$, and this bound is tight. Note also that the $k$-power domination number of the only exception to the general bound is $\gamma_{\mathrm{P}}\left(K_{k+2, k+2}\right)=2=\frac{n+2}{k+3}$. A proof of Theorem 2.1 is presented in section 5. First we construct a special family of graphs in section 3 . In order to present a proof of Theorem 2.1, we shall need the concept of an $(A, B)$-configuration in a graph which we define in section 4 . In that section, we establish important properties of $(A, B)$-configurations that we shall need in the proof of our main result. Throughout the rest of the paper $k$ will denote a positive integer.

3. The family $\mathcal{F}_{k}$. In this section, we define a special family $\mathcal{F}_{k}$ of graphs as follows (see Figure 3.1).

Definition 3.1 (the family $\mathcal{F}_{k}$ ). For $k \geq 2$, let $F_{k}$ be a graph on $2 k+5$ vertices constructed as follows. Let two independent sets $X_{1}$ and $X_{2}$ of order $k+1$ form together with three vertices $u, x$, and $y$ the set of vertices of $F_{k}$. Join by an edge every vertex of $X_{2}$ to $x$, and every vertex of $X_{1}$ to $y$. Join $u$ to any $k+2$ vertices in $X_{1} \cup X_{2}$. Finally, add edges arbitrarily between $X_{1}$ and $X_{2}$ so that every vertex of $X_{1} \cup X_{2}$ reaches degree $k+2$, (i.e., for $i \in\{1,2\}$, every vertex in $X_{i}$ is adjacent to 


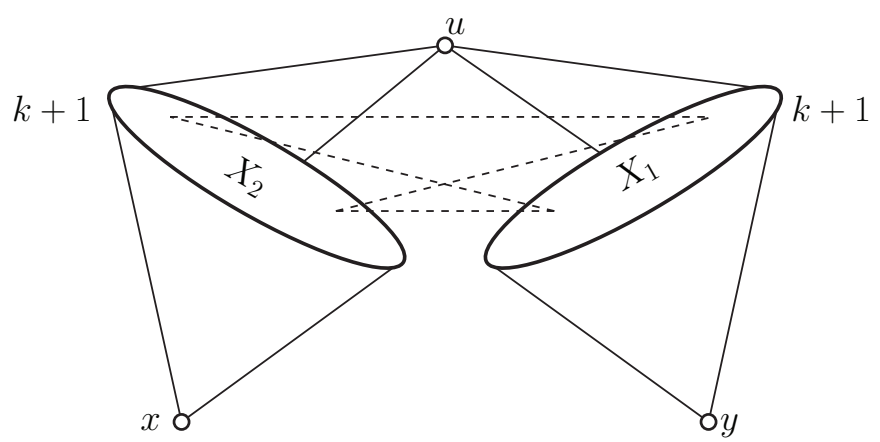

FIG. 3.1. A graph of the family $\mathcal{F}_{k}$.

$k+1$ vertices in $X_{3-i} \cup\{u\}$ and to either $x$ or $\left.y\right)$. Note that this is possible only if $k$ is even and the neighbors of $u$ are equally distributed among $X_{1}$ and $X_{2}$. Thereafter, every vertex in $V\left(F_{k}\right)$ is of degree $k+2$ except for $x$ and $y$, which are of degree $k+1$. We call the vertex $u$ the focal vertex of $F_{k}$. For $k \geq 2$, let $\mathcal{F}_{k}$ be the family of all such graphs $F_{k}$.

DEFINITION 3.2 (a subgraph of type- $\mathcal{F}_{k}$ ). If a graph $G$ has a subgraph, not necessarily induced, that is isomorphic to a graph in the family $\mathcal{F}_{k}$, then we call such a subgraph of $G$ a subgraph of type- $\mathcal{F}_{k}$.

4. $(\boldsymbol{A}, \boldsymbol{B})$-configurations. In this section, we define an $(A, B)$-configuration in a graph, a key concept to prove our main result, namely, Theorem 2.1.

Definition $4.1((A, B)$-configurations). Let $G$ be a connected $(k+2)$-regular graph. For subsets $A$ and $B$ of vertices in $G$, we define the subgraph $G[A \cup B]$ of $G$ induced by the sets $A \cup B$ to be an $(A, B)$-configuration if the following four properties hold:

(P1) $|A| \in\{k+1, k+2\}$.

(P2) $B=N(A) \backslash A$.

(P3) $d_{A}(v)=k+1$ for each vertex $v \in B$.

(P4) $B$ is an independent set.

Note that if a graph contains a subgraph of type- $\mathcal{F}_{k}$, then it contains at least two ( $A, B$ )-configurations: one where $A=X_{2} \cup\{u\}$ and $B=X_{1} \cup\{x\}$, and another where $A^{\prime}=X_{1} \cup\{u\}$ and $B^{\prime}=X_{2} \cup\{y\}$. Also, a subgraph isomorphic to $K_{k+2, k+2}-e$, say, with bipartition $V=(X \cup\{x\}, Y \cup\{y\})$ and $e=x y$, contains two $(A, B)$ configurations: one where $A=X$ and $B=Y \cup\{y\}$, and another where $A^{\prime}=Y$ and $B^{\prime}=X \cup\{x\}$. These two examples will prove key in the following.

We next establish three additional properties of an $(A, B)$-configuration that will prove to be useful.

Lemma 4.2. Let $G$ be a connected $(k+2)$-regular graph and let $A$ and $B$ be subsets of vertices in $G$ that induce an $(A, B)$-configuration. Then the following properties hold:

$(\mathrm{P} 5) d_{B}(v) \geq 1$ for each vertex $v \in A$.

(P6) If $k$ is odd, then $|A|=k+1$.

(P7) $|B| \leq k+2$.

Proof. (P5) Let $v \in A$. By the regularity of $G$, we have $d(v)=k+2$. Further, since $|A| \leq k+2$ by property (P1), we note that $d_{A}(v) \leq|A|-1 \leq k+1$. By property (P2), every neighbor of $v$ not in $A$ belongs to $B$, and so $k+2=d(v)=$ $d_{A}(v)+d_{B}(v) \leq k+1+d_{B}(v)$, implying that $d_{B}(v) \geq 1$. 
(P6) Suppose that $k$ is odd but $|A|=k+2$. We reach a contradiction by double counting the number of edges in $[A, B]$ as follows. By property $(\mathrm{P} 3)$, we have that

$$
|[A, B]|=|B| \times(k+1),
$$

while summing the degrees of vertices in $A$, we have by property (P2) that

$$
\begin{aligned}
|[A, B]| & =|A| \times(k+2)-(2 \times|[A, A]|) \\
& =(k+2)^{2}-2|[A, A]| .
\end{aligned}
$$

Hence,

$$
(k+1)|B|=(k+2)^{2}-2|[A, A]| .
$$

By assumption, $k$ is odd, and so $(k+1)|B|$ is even, whereas $(k+2)^{2}-2|[A, A]|$ is odd. Hence, the left hand side of equality (4.1) is odd, while the right hand side is even, a contradiction. Therefore if $k$ is odd, and then $|A| \neq k+2$, implying by property (P1) that $|A|=k+1$. This proves property (P6).

(P7) To prove property (P7), we consider two cases depending on the order of $|A|$. Suppose that $|A|=k+1$. Then by property (P3), we have that $d_{A}(v)=k+1$ for each vertex $v \in B$, implying that $N_{A}(v)=A$ for each $v \in B$. Hence, if $y \in A$, then $B \subseteq N(y)$, and so $k+2=d(y)=d_{A}(y)+d_{B}(y)=d_{A}(y)+|B|$, implying that $|B|=k+2-d_{A}(y) \leq k+2$. Hence, we may assume that $|A|=k+2$, for otherwise $|B| \leq k+2$, as desired. But then equality (4.1) holds as shown in the proof of property (P6). If $k$ is odd, then as before the left hand side of equality (4.1) is even, while the right hand side is odd, a contradiction. Hence, $k$ is even. Thus, the right hand side of equality (4.1) is even, implying that $|B|$ is even since $k+1$ is odd. Further, by equality (4.1), we have that

$$
\begin{aligned}
|B| & =\frac{(k+2)^{2}}{k+1}-\frac{2|[A, A]|}{k+1} \\
& \leq \frac{(k+2)^{2}}{k+1} \\
& =k+3+\frac{1}{k+1} \\
& \leq k+3
\end{aligned}
$$

as $k>0$. However, $|B|$ is even and $k+3$ is odd, implying that $|B| \leq k+2$, as desired.

Throughout the rest of this section 4 on $(A, B)$-configurations, we let $G=(V, E)$ be a connected $(k+2)$-regular graph of order at least $2 k+4$. Further, we let $A$ and $B$ be subsets of vertices in $G$ that induce an $(A, B)$-configuration and let $A^{\prime}$ and $B^{\prime}$ be subsets of vertices in $G$ that induce an $\left(A^{\prime}, B^{\prime}\right)$-configuration, where $A \neq A^{\prime}$. We proceed further with seven key lemmas about structural properties of $(A, B)$ configurations.

Lemma 4.3. The following properties hold in the graph $G$ :

(a) If $\left|A \cap A^{\prime}\right|>1$, then $B \cup B^{\prime} \subseteq A \cup A^{\prime} \cup\left(B \cap B^{\prime}\right)$.

(b) If $\left|A \cap A^{\prime}\right|>1$, then $B \cap B^{\prime} \neq \emptyset$.

(c) If $\left|A \cap A^{\prime}\right| \geq 1$, then $\left|A \cap A^{\prime}\right| \in\{1, k, k+1\}$.

Proof. (a) Suppose $\left|A \cap A^{\prime}\right|>1$. Let $u$ and $v$ be two distinct vertices in $A \cap A^{\prime}$. By properties (P1) and (P3), every vertex in $B$ is adjacent to at least one of $u$ and $v$, 
implying that $B \subseteq N(u) \cup N(v)$. Analogously, $B^{\prime} \subseteq N(u) \cup N(v)$. Since $\{u, v\} \subseteq A$, we note that $N(u) \cup N(v) \subseteq A \cup B$ by property (P2). Analogously, since $\{u, v\} \subseteq A^{\prime}$ we have that $N(u) \cup N(v) \subseteq A^{\prime} \cup B^{\prime}$. Therefore, $B \cup B^{\prime} \subseteq N(u) \cup N(v) \subseteq(A \cup B) \cap\left(A^{\prime} \cup B^{\prime}\right)$. Part (a) now follows from the observation that $(A \cup B) \cap\left(A^{\prime} \cup B^{\prime}\right) \subseteq A \cup A^{\prime} \cup\left(B \cap B^{\prime}\right)$.

(b) Suppose $\left|A \cap A^{\prime}\right|>1$. We show that $B \cap B^{\prime} \neq \emptyset$. For the sake of contradiction, assume that $B \cap B^{\prime}=\emptyset$. Then by part (a), $B \cup B^{\prime} \subseteq A \cup A^{\prime}$. Thus by property (P2), $N\left(A \cup A^{\prime}\right) \subseteq A \cup A^{\prime} \cup\left(B \cup B^{\prime}\right) \subseteq A \cup A^{\prime}$. Hence, the connectivity of $G$ implies that the vertices of $A \cup A^{\prime}$ induce the whole graph, i.e., $G=G\left[A \cup A^{\prime}\right]$. Further, by property (P1) and since $\left|A \cap A^{\prime}\right| \geq 2$ by assumption, we have that $|V|=\left|A \cup A^{\prime}\right|=$ $|A|+\left|A^{\prime}\right|-\left|A \cap A^{\prime}\right| \leq(k+2)+(k+2)-2=2 k+2$. This contradicts our assumption that $|V| \geq 2 k+4$. Therefore, $B \cap B^{\prime} \neq \emptyset$, establishing part (b).

(c) Suppose $\left|A \cap A^{\prime}\right| \geq 1$. If $\left|A \cap A^{\prime}\right|=1$, then part (c) is immediate. Hence, we may assume that $\left|A \cap A^{\prime}\right| \geq 2$, for otherwise there is nothing to prove. By part (b), $B \cap B^{\prime} \neq \emptyset$. Let $x \in B \cap B^{\prime}$. By property (P3), $d_{A}(x)=k+1$ and $d_{A^{\prime}}(x)=k+1$. Hence,

$$
k+2=d(x) \geq d_{A \cup A^{\prime}}(x)=d_{A}(x)+d_{A^{\prime}}(x)-d_{A \cap A^{\prime}}(x)=2(k+1)-d_{A \cap A^{\prime}}(x),
$$

and so $d_{A \cap A^{\prime}}(x) \geq k$, implying that $\left|A \cap A^{\prime}\right| \geq k$. Further, since $A \neq A^{\prime}$, property (P1) implies that $\left|A \cap A^{\prime}\right| \leq k+1$. Hence, $\left|A \cap A^{\prime}\right| \in\{k, k+1\}$. This proves part (c).

Lemma 4.4. If $\left|A \cap A^{\prime}\right|=k$, where $k \geq 2$, then $G=K_{k+2, k+2}$.

Proof. Suppose $\left|A \cap A^{\prime}\right|=k$, where $k \geq 2$. By Lemma 4.3(b), $B \cap B^{\prime} \neq \emptyset$. Let $x \in B \cap B^{\prime}$. By property (P3), $d_{A}(x)=k+1$ and $d_{A^{\prime}}(x)=k+1$. Hence,

$$
k+2=d(x) \geq d_{A \cup A^{\prime}}(x)=d_{A}(x)+d_{A^{\prime}}(x)-d_{A \cap A^{\prime}}(x)=2(k+1)-d_{A \cap A^{\prime}}(x),
$$

and so $d_{A \cap A^{\prime}}(x) \geq k$. However, since $\left|A \cap A^{\prime}\right|=k$, we note that $d_{A \cap A^{\prime}}(x) \leq k$. Consequently, $d_{A \cap A^{\prime}}(x)=k$. Hence, we must have equality throughout the inequality chain (4.2). In particular, $d(x)=d_{A \cup A^{\prime}}(x)$. Since $d_{A}(x)=d_{A^{\prime}}(x)=k+1$ and $d_{A \cap A^{\prime}}(x)=k$, we have that $d_{A \backslash A^{\prime}}(x)=1$ and $d_{A^{\prime} \backslash A}(x)=1$. Since $d(x)=k+2$, we note that $x$ has no further neighbors in $G$. Since $x$ is an arbitrary vertex in $B \cap B^{\prime}$, we therefore have that every vertex in $B \cap B^{\prime}$ is adjacent to every vertex in $A \cap A^{\prime}$ and to exactly one vertex in each of the sets $A \backslash A^{\prime}$ and $A^{\prime} \backslash A$. In particular, each vertex in $B \cap B^{\prime}$ has no neighbor in $V \backslash\left(A \cup A^{\prime}\right)$. Thus, $N\left(B \cap B^{\prime}\right) \subseteq A \cup A^{\prime}$. By Lemma 4.3(a), $B \cup B^{\prime} \subseteq A \cup A^{\prime} \cup\left(B \cap B^{\prime}\right)$. Therefore, by the connectivity of $G$ we deduce that the vertices of $A \cup A^{\prime} \cup\left(B \cap B^{\prime}\right)$ induce the whole graph, i.e., $G=G\left[A \cup A^{\prime} \cup\left(B \cap B^{\prime}\right)\right]$.

We consider once again the vertex $x \in B \cap B^{\prime}$. As observed earlier, $d_{A \backslash A^{\prime}}(x)=1$ and $d_{A^{\prime} \backslash A}(x)=1$. Let $u$ be the unique vertex in $N_{A \backslash A^{\prime}}(x)$ and let $v$ be the unique vertex in $N_{A^{\prime} \backslash A}(x)$. If $u \in B^{\prime}$, then there is an edge joining two vertices in $B^{\prime}$, namely the edge $u x$, contradicting the independence of the set $B^{\prime}$ by property (P4). Hence, $u \notin B^{\prime}$, implying that $u$ is not adjacent to any vertex in $A^{\prime}$. However, by Lemma 4.3(a), $B \cup B^{\prime} \subseteq A \cup A^{\prime} \cup\left(B \cap B^{\prime}\right)$. Hence, since $N(u) \cap\left(A^{\prime} \cup\{u\}\right)=\emptyset$ and $N(u) \subseteq A \cup B$, we have that $N(u) \subseteq\left(A \backslash\left(A^{\prime} \cup\{u\}\right)\right) \cup\left(B \cap B^{\prime}\right)$. However, $\left|A \backslash\left(A^{\prime} \cup\{u\}\right)\right| \leq 1$. Thus,

$$
k+2=d(u)=d_{A \backslash\left(A^{\prime} \cup\{u\}\right)}(u)+d_{B \cap B^{\prime}}(u) \leq 1+d_{B \cap B^{\prime}}(u),
$$

and so $d_{B \cap B^{\prime}}(u) \geq k+1$, implying that $\left|B \cap B^{\prime}\right| \geq k+1$.

Suppose that $\left|B \cap B^{\prime}\right|=k+1$. Then, $d_{B \cap B^{\prime}}(u)=k+1$ and we must have equality throughout the inequality chain (4.3). In particular, $d_{A \backslash\left(A^{\prime} \cup\{u\}\right)}(u)=1$. However, 
$|A| \leq k+2$ and $\left|A \cap A^{\prime}\right|=k$, and so $\left|A \backslash A^{\prime}\right|=2$ and $|A|=k+2$. Let $y$ be the vertex of $A \backslash A^{\prime}$ adjacent to $u$, and so $A \backslash A^{\prime}=\{u, y\}$ and $u y \in E$. Analogously, we have that $v \notin B, v$ is not adjacent to any vertex in $A, d_{B \cap B^{\prime}}(v)=k+1$, and $v$ is adjacent to a vertex, $z$, say, in $A^{\prime} \backslash A$. Thus, $A^{\prime} \backslash A=\{v, z\}$ and $v z \in E$. Hence, every vertex in $B \cap B^{\prime}$ is adjacent to the $k$ vertices in $A \cap A^{\prime}$ and to both $u$ and $v$ (and thus to no other vertices). Since $v$ is not adjacent to any vertex in $A$, we note that $v y \notin E$. Thus, $N(y) \subseteq\left(A \cap A^{\prime}\right) \cup\{u, z\}$, and so $k+2=d(y) \leq\left|A \cap A^{\prime}\right|+2=k+2$, implying that we must have equality throughout this inequality chain and therefore $N(y)=\left(A \cap A^{\prime}\right) \cup\{u, z\}$. Analogously, $N(z)=\left(A \cap A^{\prime}\right) \cup\{v, y\}$. But then each vertex in $A \cap A^{\prime}$ is adjacent to both $y$ and $z$ and to the $k+1$ vertices in $B \cap B^{\prime}$ and therefore has degree at least $k+3$, contradicting the $(k+2)$-regularity of $G$. Hence, $\left|B \cap B^{\prime}\right| \geq k+2$. Consequently, by property (P7), $\left|B \cap B^{\prime}\right|=k+2$ and $B \cap B^{\prime}=B=B^{\prime}$.

We show next that $|A|=k+1$. Assume, to the contrary, that $|A|=k+2$. Then, $\left|A \backslash A^{\prime}\right|=2$. Let $y$ denote the vertex in $A \backslash A^{\prime}$ distinct from $u$, and so $A \backslash A^{\prime}=\{u, y\}$. Since $\{u, y\} \subseteq A$, we note by property (P2) that neither $u$ nor $y$ belong to $B$. Thus since $B=B^{\prime}$, neither $u$ nor $y$ belong to $B^{\prime}$, implying that $u$ and $y$ are not adjacent to any vertex in $A^{\prime}$. Every vertex in $B \cap B^{\prime}$ is adjacent to the $k$ vertices in $A \cap A^{\prime}$ and to exactly one of $u$ and $y$. Hence, defining $\delta_{E}(e)=1$ when $e \in E$ and 0 otherwise, we have that

$$
2 k+4=d(u)+d(y)=\left|\left[\{u, y\}, B \cap B^{\prime}\right]\right|+2 \delta_{E}(u y)=\left|B \cap B^{\prime}\right|+2 \delta_{E}(u y) \leq k+4,
$$

and so $k \leq 0$, contradicting our assumption that $k \geq 2$. Hence, $|A|=k+1$. Analogously, $\left|A^{\prime}\right|=k+1$. Thus, $A \backslash A^{\prime}=\{u\}$ and $A^{\prime} \backslash A=\{v\}$. From our earlier observations, $\left|B \cap B^{\prime}\right|=k+2$ and every vertex in $B \cap B^{\prime}$ is adjacent to the $k$ vertices in $A \cap A^{\prime}$ and to both $u$ and $v$, implying by the $(k+2)$-regularity of $G$ that $G=K_{k+2, k+2} . \quad$ प

Lemma 4.5. If $\left|A \cap A^{\prime}\right|=k+1$, where $k \geq 1$, then $\gamma_{\mathrm{P}, k}(G)=1$.

Proof. Suppose $\left|A \cap A^{\prime}\right|=k+1$, where $k \geq 1$. In particular, $\left|A \cap A^{\prime}\right| \geq 2$. By assumption, $A \neq A^{\prime}$. Renaming the sets $A$ and $A^{\prime}$, if necessary, we may assume that there exists a vertex $x \in A \backslash A^{\prime}$. By property (P1), $|A| \leq k+2$, and so $|A|=k+2$ and $A=\left(A \cap A^{\prime}\right) \cup\{x\}$. By property (P5), $d_{B}(x) \geq 1$. Let $y \in N_{B}(x)$.

Suppose $y \notin B \cap B^{\prime}$. Since $y \in B$, by property (P2), $y \notin A$. Hence, by Lemma 4.3(a) we have that $y \in A^{\prime} \backslash A$. This is true for every neighbor of $x$ in $B \backslash B^{\prime}$. Since $x \notin A^{\prime}$, we note that $x \in B^{\prime}$. By property (P4), the set $B^{\prime}$ is an independent set, and so $x$ is not adjacent to any vertex in $B \cap B^{\prime}$. Since $x \in A$, we have that $N(x) \subseteq A \cup B$ and every neighbor of $x$ in $B$ belongs to the set $A^{\prime} \backslash A$. We deduce, therefore, that $N(x) \subseteq\left(A \cap A^{\prime}\right) \cup\left(A^{\prime} \backslash A\right)=A^{\prime}$, and so $k+2=d(x)=d_{A^{\prime}}(x) \leq\left|A^{\prime}\right| \leq k+2$. Hence, we must have equality throughout this inequality chain. In particular, $d_{A^{\prime}}(x)=k+2$. However, $x \in B^{\prime}$, and so by property (P3), $d_{A^{\prime}}(x)=k+1$, a contradiction. Therefore, $y \in B \cap B^{\prime}$. This is true for every neighbor of $x$ in $B$, and so $N_{B}(x) \subseteq B \cap B^{\prime}$.

By property (P3), $d_{A}(y)=k+1$, and so the vertex $y$ is adjacent to exactly $k$ vertices in $A \cap A^{\prime}$. Further, $d_{A^{\prime}}(y)=k+1$, implying that $y$ is adjacent to a vertex in $A^{\prime} \backslash A$, say $z$. Since $\left|A^{\prime}\right| \leq k+2$, we therefore have that $\left|A^{\prime}\right|=k+2$ and $A^{\prime} \backslash A=\{z\}$. By property (P4), the set $B$ is an independent set. Hence, since $z$ is adjacent to a vertex in $B$, namely, to the vertex $y$, we note that $z \notin B$. Thus, $z$ is not adjacent to any vertex of $A$, and so $x z \notin E$ and $z$ is not adjacent to any vertex in $A \cap A^{\prime}$. An analogous argument as with the vertex $x \in A \backslash A^{\prime}$ shows that for the vertex $z \in A^{\prime} \backslash A$, 
we have $N_{B^{\prime}}(z) \subseteq B \cap B^{\prime}$. Since $\left(A^{\prime} \cup A\right) \backslash\{z\}=A$ and $z$ is adjacent to no vertex in $A$, we have that $N(z) \subseteq B \cap B^{\prime}$. By property (P7), $\left|B \cap B^{\prime}\right| \leq k+2$, implying by the $(k+2)$-regularity of $G$ that $N(z)=B \cap B^{\prime},\left|B \cap B^{\prime}\right|=k+2$, and $B=B^{\prime}=B \cap B^{\prime}$. Analogously, $N(x)=B \cap B^{\prime}$. Thus every vertex in $B \cap B^{\prime}$ is adjacent to exactly $k$ vertices in $A \cap A^{\prime}$ and to both $x$ and $z$. Hence, $N\left(B \cap B^{\prime}\right) \subseteq A \cup A^{\prime}$. By Lemma 4.3(a), $B \cup B^{\prime} \subseteq A \cup A^{\prime} \cup\left(B \cap B^{\prime}\right)$. Therefore, by the connectivity of $G$ we deduce that the vertices of $A \cup A^{\prime} \cup\left(B \cap B^{\prime}\right)$ induce the whole graph, i.e., $G=G\left[A \cup A^{\prime} \cup\left(B \cap B^{\prime}\right)\right]$.

We now double count the edges in the set $\left[A \cap A^{\prime}, B \cap B^{\prime}\right]$. First counting the edges emanating from $B \cap B^{\prime}$, we have

$$
\left|\left[A \cap A^{\prime}, B \cap B^{\prime}\right]\right|=\left|B \cap B^{\prime}\right| \times k=k(k+2) .
$$

Counting the edges emanating from $A \cap A^{\prime}$, we have

$$
\begin{aligned}
\left|\left[A \cap A^{\prime}, B \cap B^{\prime}\right]\right| & =\left|A \cap A^{\prime}\right| \times(k+2)-\left(2 \times\left|\left[A \cap A^{\prime}, A \cap A^{\prime}\right]\right|\right) \\
& =(k+1)(k+2)-\sum_{u \in A \cap A^{\prime}} d_{A \cap A^{\prime}}(u) .
\end{aligned}
$$

Therefore,

$$
\sum_{u \in A \cap A^{\prime}} d_{A \cap A^{\prime}}(u)=k+2 .
$$

Since $\left|A \cap A^{\prime}\right|=k+1$, there exists a vertex $v \in A \cap A^{\prime}$ with $d_{A \cap A^{\prime}}(v) \geq 2$. Let $u$ and $w$ be two neighbors of $v$ in $A \cap A^{\prime}$. We show that $\{v\}$ is a kPD-set in $G$. As observed earlier, every vertex in $B \cap B^{\prime}$ is adjacent to exactly $k$ vertices in $A \cap A^{\prime}$. Equivalently, every vertex in $B \cap B^{\prime}$ is not adjacent to exactly one vertex in $A \cap A^{\prime}$. Let $u^{\prime}$ and $w^{\prime}$ be the vertices in $B \cap B^{\prime}$ not adjacent to $u$ and $w$, respectively. Thus, $u^{\prime}$ is adjacent to every vertex of $A \cap A^{\prime}$ different from $u$ (among which $w$, implying $u^{\prime} \neq w^{\prime}$ ), while $w^{\prime}$ is adjacent to every vertex of $A \cap A^{\prime}$ different from $w$. In particular, since $v w^{\prime} \in E$, we have that $w^{\prime} \in \mathcal{P}^{0}(\{v\})$. Since $u w^{\prime} \in E$, we note that $\left|N\left[w^{\prime}\right] \backslash \mathcal{P}^{0}(\{v\})\right| \leq d\left(w^{\prime}\right)-2=k$, and so $N\left[w^{\prime}\right] \subseteq \mathcal{P}^{1}(\{v\})$. In particular, $\{x, z\} \subseteq \mathcal{P}^{1}(\{v\})$. Analogously, $u^{\prime} \in \mathcal{P}^{0}(\{v\})$ and $N\left[u^{\prime}\right] \subseteq \mathcal{P}^{1}(\{v\})$. Hence, $\left|N[x] \backslash \mathcal{P}^{1}(\{v\})\right| \leq\left|\left(B \cap B^{\prime}\right) \backslash\left\{u^{\prime}, w^{\prime}\right\}\right|=k$, and so $N[x] \subseteq \mathcal{P}^{2}(\{v\})$. Therefore, $V=N[x] \cup N\left[w^{\prime}\right] \cup\{w\} \subseteq \mathcal{P}^{2}(\{v\})$, and so $\{v\}$ is a kPD-set in $G$. Thus, $\gamma_{\mathrm{P}, k}(G)=1$.

Lemma 4.6. If $\left|A \cap A^{\prime}\right|=1$, then either $G\left[A \cup A^{\prime} \cup B \cup B^{\prime}\right]$ is a subgraph of $G$ of type- $\mathcal{F}_{k}$ or $k=1$ and $G=K_{3,3}$.

Proof. Suppose $\left|A \cap A^{\prime}\right|=1$ and let $A \cap A^{\prime}=\{u\}$. We proceed further with a series of four claims.

Claim I. If $A^{\prime} \cap B=\emptyset$ or $A \cap B^{\prime}=\emptyset$, then $G=K_{3,3}$.

Proof. Suppose that $A^{\prime} \cap B=\emptyset$. By property (P5), the vertex $u$ is adjacent to a vertex in $B$, say, $v$. Since $v \notin A^{\prime}$ and $v$ is adjacent to a vertex of $A^{\prime}$, we note that $v \in B^{\prime}$, and so $v \in B \cap B^{\prime}$. By property (P3), $d_{A}(v)=d_{A^{\prime}}(v)=k+1$. Hence, $k+2=d(v) \geq d_{A \cup A^{\prime}}(v)=d_{A}(v)+d_{A^{\prime}}(v)-d_{A \cap A^{\prime}}(v)=2(k+1)-1=2 k+1$, and so $k \leq 1$. Consequently, $k=1$, and therefore by property (P6) we have that $|A|=\left|A^{\prime}\right|=k+1=2$. Thus by property (P3), every vertex in $B$ is adjacent to both vertices in $A$. In particular, every vertex in $B$ is adjacent to the vertex $u$. Since $u \in A^{\prime}$ and $A^{\prime} \cap B=\emptyset$, this implies that $B \subseteq B^{\prime}$ and every vertex in $B$ is adjacent to both vertices in $A^{\prime}$. Analogously, $B^{\prime} \subseteq B$. Consequently, $B=B^{\prime}=B \cap B^{\prime}$. Since $k=1$, we note that $G$ is a 3 -regular graph. Therefore, since each vertex in $B \cap B^{\prime}$ is adjacent to all three vertices in $A \cup A^{\prime}$, we have that $N(v)=A \cup A^{\prime}$ for each vertex 
$v \in B \cap B^{\prime}$. The connectivity of $G$ implies that the vertices of $A \cup A^{\prime} \cup\left(B \cap B^{\prime}\right)$ induce the whole graph, i.e., $G=G\left[A \cup A^{\prime} \cup\left(B \cap B^{\prime}\right)\right]$. Let $\left|B \cap B^{\prime}\right|=\ell$. By property (P7), we note that $1 \leq \ell \leq 3$ since $k=1$. Since $G$ is 3-regular and every vertex in $B \cap B^{\prime}$ is adjacent to every vertex in $A \cup A^{\prime}$, the set $\left[A \cup A^{\prime}, B \cap B^{\prime}\right]$ is full, implying that the graph $G\left[A \cup A^{\prime}\right]$ is a $(3-\ell)$-regular graph. Hence, since $\left|A \cup A^{\prime}\right|=3$, we note that $3-\ell$ is even, and so $\ell \in\{1,3\}$. If $\ell=1$, then $G=K_{4}$ and so $|V|=4$, contradicting the assumption that $|V| \geq 2 k+4=6$. Therefore, $\ell=3$ and $G=K_{3,3}$. Analogously, if $A \cap B^{\prime}=\emptyset$, then $G=K_{3,3}$.

By Claim I, we may assume that $A^{\prime} \cap B \neq \emptyset$ and $B^{\prime} \cap A \neq \emptyset$, for otherwise $G=K_{3,3}$ and we are done.

Claim II. $\left|A \cap B^{\prime}\right| \geq k$ and $\left|A^{\prime} \cap B\right| \geq k$.

Proof. By assumption, $A^{\prime} \cap B \neq \emptyset$. Let $v \in B \cap A^{\prime}$. By property (P3), $d_{A}(v)=$ $k+1$, and by property (P2), $N(v) \subseteq A^{\prime} \cup B^{\prime}$. Hence, since $\left|A \cap A^{\prime}\right|=1$ and the vertex $v$ is adjacent to at least $k$ vertices of $A \backslash A^{\prime}(=A \backslash\{u\})$, we have that $\left|A \cap B^{\prime}\right| \geq k$. Analogously, $\left|A^{\prime} \cap B\right| \geq k$.

Claim III. $A \backslash\left(A^{\prime} \cup B^{\prime}\right)=\emptyset$ and $A^{\prime} \backslash(A \cup B)=\emptyset$.

Proof. For the sake of contradiction, assume that $\left|A \backslash\left(A^{\prime} \cup B^{\prime}\right)\right| \geq 1$. By Claim II, $\left|A \cap B^{\prime}\right| \geq k$. Thus since $\left|A \cap A^{\prime}\right|=1$ and $A^{\prime} \cap B^{\prime}=\emptyset$, we have that $k+2 \geq|A|=$ $\left|A \backslash\left(A^{\prime} \cup B^{\prime}\right)\right|+\left|A \cap B^{\prime}\right|+\left|A \cap A^{\prime}\right| \geq 1+k+1=k+2$. Hence, we must have equality throughout this inequality chain, implying that $|A|=k+2,\left|A \backslash\left(A^{\prime} \cup B^{\prime}\right)\right|=1$, and $\left|A \cap B^{\prime}\right|=k$. In particular since $|A|=k+2$, we have by property (P6) that $k$ is even, and so $k \geq 2$. Let $A \backslash\left(A^{\prime} \cup B^{\prime}\right)=\{x\}$, and so $A=\{u, x\} \cup\left(A \cap B^{\prime}\right)$. Since the vertex $x$ has no neighbors in $A^{\prime}$, we note that all $k+2$ neighbors of $x$ belong to the set $\left(A \backslash A^{\prime}\right) \cup\left(B \backslash A^{\prime}\right)$. Thus, since $|A|=k+2$ and since $|B| \leq k+2$ by property (P7), we have that

$$
\begin{aligned}
k+2 & =d(x) \\
& =d_{A \backslash A^{\prime}}(x)+d_{B \backslash A^{\prime}}(x) \\
& \leq|A \backslash\{u, x\}|+\left|B \backslash A^{\prime}\right| \\
& =k+|B|-\left|A^{\prime} \cap B\right| \\
& \leq k+(k+2)-k \\
& =k+2 .
\end{aligned}
$$

Consequently, we must have equality throughout the above inequality chain, implying that $|A \backslash\{u, x\}|=k,\left|A^{\prime} \cap B\right|=k$ and $\left|B \backslash A^{\prime}\right|=2$. Further, $A \backslash\{u, x\}=$ $A \cap B^{\prime} \subseteq N(x)$. Let $B \backslash A^{\prime}=\{y, z\}$. By property (P3), $d_{A}(y)=k+1$. Since $k \geq 2$, the vertex $y$ therefore has a neighbor in $A \cap B^{\prime}$, say, $w$. Since $w \in B^{\prime}$, by property (P3), $d_{A^{\prime}}(w)=k+1$. However, $w$ is adjacent to $x$ and $y$, neither of which belong to $A^{\prime}$. Hence, $d(w) \geq d_{A^{\prime}}(w)+2 \geq k+3$, a contradiction. Therefore, $A \backslash\left(A^{\prime} \cup B^{\prime}\right)=\emptyset$. Analogously, $A^{\prime} \backslash(A \cup B)=\emptyset$. This completes the proof of Claim III.

By Claim III, $A \backslash\left(A^{\prime} \cup B^{\prime}\right)=\emptyset$, and so $A \subseteq A^{\prime} \cup B^{\prime}$. Further, by Claim III, $A^{\prime} \backslash(A \cup B)=\emptyset$, and so $A^{\prime} \subseteq A \cup B$.

Claim IV. $B^{\prime} \backslash(A \cup B) \neq \emptyset$ and $B \backslash\left(A^{\prime} \cup B^{\prime}\right) \neq \emptyset$.

Proof. Suppose that $B^{\prime} \subseteq A \cup B$. By Claim II, $\left|A^{\prime} \cap B\right| \geq k$. Let $v \in A^{\prime} \cap B$. Since $v \in A^{\prime}$, by property (P2) we note that $N(v) \subseteq A^{\prime} \cup B^{\prime}$. As observed earlier, $A^{\prime} \subseteq A \cup B$. By assumption, $B^{\prime} \subseteq A \cup B$. Hence, $A \cup B^{\prime} \subseteq A \cup B$, and so $N(v) \subseteq A \cup B$ and $d_{A \cup B}(v)=d(v)=k+2$. However, $v \in B$, and by property (P4) the set $B$ 
is an independent set, implying that $d_{B}(v)=0$ and $d_{A}(v)=k+2$, contradicting property $(\mathrm{P} 3)$. Hence, $B^{\prime} \backslash(A \cup B) \neq \emptyset$. Analogously, $B \backslash\left(A^{\prime} \cup B^{\prime}\right) \neq \emptyset$.

By Claim IV, $B^{\prime} \backslash(A \cup B) \neq \emptyset$ and $B \backslash\left(A^{\prime} \cup B^{\prime}\right) \neq \emptyset$. Let $x \in B \backslash\left(A^{\prime} \cup B^{\prime}\right)$ and let $y \in B^{\prime} \backslash(A \cup B)$. Set $X_{1}=A^{\prime} \backslash\{u\}$ and $X_{2}=A \backslash\{u\}$. Since $A \cap A^{\prime}=\{u\}$ and $A \subseteq A^{\prime} \cup B^{\prime}$ while $A^{\prime} \subseteq A \cup B$, we note that $X_{1}=A^{\prime} \cap B$ and $X_{2}=A \cap B^{\prime}$. Since $x \in B$, by property (P3) we have $d_{A}(x)=k+1$. However, $x \notin A^{\prime} \cup B^{\prime}$, and so $N(x) \cap A^{\prime}=\emptyset$. Hence, $N_{A}(x) \subseteq A \backslash\{u\}=X_{2}$, implying that $\left|X_{2}\right|=k+1$ and $x$ is adjacent to every vertex of $X_{2}$. Since $\{y\} \cup X_{2} \subseteq B^{\prime}$ and $\left|B^{\prime}\right| \leq k+2$ by property (P7), we have that $\left|B^{\prime}\right|=k+2$ and $B^{\prime}=\{y\} \cup X_{2}$. Analogously, $\left|X_{1}\right|=k+1,|B|=k+2$, $B=\{x\} \cup X_{1}$, and $y$ is adjacent to every vertex in $X_{1}$. In particular, we have that $\{u, x, y\} \cup X_{1} \cup X_{2}$ is a partition of the set $A \cup A^{\prime} \cup B \cup B^{\prime}$. The vertex $u$ is adjacent to neither $x$ nor $y$, and so $N(u) \subseteq X_{1} \cup X_{2}$. By the $(k+2)$-regularity of $G$, the vertex $u$ therefore has $k+2$ neighbors in $X_{1} \cup X_{2}$. Since $X_{1} \subset B$ and $A=X_{2} \cup\{u\}$, by property (P3) every vertex in $X_{1}$ has $k+1$ neighbors in $X_{2} \cup\{u\}$. Analogously, every vertex in $X_{2}$ has $k+1$ neighbors in $X_{1} \cup\{u\}$. Therefore, $G\left[A \cup A^{\prime} \cup B \cup B^{\prime}\right]$ contains a subgraph of type- $\mathcal{F}_{k}$. We remark that if $x y \notin E$, then this subgraph is an induced subgraph of $G$. This completes the proof of Lemma 4.6.

Lemma 4.7. If $\left|B \cap B^{\prime}\right| \geq 1$, then $\left|A \cap A^{\prime}\right| \geq k$.

Proof. Suppose $B \cap B^{\prime} \neq \emptyset$. Let $u \in B \cap B^{\prime}$. By property (P3), $d_{A}(u)=k+1$ and $d_{A^{\prime}}(u)=k+1$. Hence, $k+2=d(u) \geq d_{A \cup A^{\prime}}(u)=d_{A}(u)+d_{A^{\prime}}(u)-d_{A \cap A^{\prime}}(u)=$ $2(k+1)-d_{A \cap A^{\prime}}(u)$, and so $d_{A \cap A^{\prime}}(u) \geq k$. $\quad$

LEMmA 4.8. If $A \cap A^{\prime}=\emptyset$ and $A \cap B^{\prime} \neq \emptyset$, then the graph $K_{k+2, k+2}-e$ is a spanning subgraph of $G\left[A \cup A^{\prime} \cup B \cup B^{\prime}\right]$.

Proof. Suppose $A \cap A^{\prime}=\emptyset$ and $A \cap B^{\prime} \neq \emptyset$. Let $x \in A \cap B^{\prime}$. Since $x \in B^{\prime}$, by property (P3), $d_{A^{\prime}}(x)=k+1$. Since $x \in A$, by property (P2), $N(x) \subseteq A \cup B$. By assumption, $A \cap A^{\prime}=\emptyset$, and so the $k+1$ neighbors of $x$ in $A^{\prime}$ all belong to $B$, implying that $\left|A^{\prime} \cap B\right| \geq k+1$. Thus, since $A^{\prime} \cap B \neq \emptyset$, analogously we have that $\left|A \cap B^{\prime}\right| \geq k+1$.

Suppose $A \backslash B^{\prime} \neq \emptyset$. Let $u \in A \backslash B^{\prime}$ and let $v \in A^{\prime} \cap B$. Since $u \notin A^{\prime} \cup B^{\prime}$ and $v \in A^{\prime}$, we note that $u v \notin E$. However, $v \in B$ and $d_{A}(v)=k+1$, implying that $N_{A}(v) \subseteq A \cap B^{\prime} \subseteq A \backslash\{u\}$. Thus, $k+1=d_{A}(v) \leq\left|A \cap B^{\prime}\right| \leq|A|-1 \leq$ $k+1$. Hence, we must have equality throughout this inequality chain, implying that $N_{A}(v)=A \cap B^{\prime}=A \backslash\{u\},\left|A \cap B^{\prime}\right|=k+1$, and $|A|=k+2$. Since $v$ is an arbitrary vertex in $A^{\prime} \cap B$, we have that every vertex in $A^{\prime} \cap B$ is adjacent to every vertex in $A \cap B^{\prime}$, that is, $\left[A^{\prime} \cap B, A \cap B^{\prime}\right]$ is full. By property (P7), $|B| \leq k+2$. Hence, $\left|B \backslash A^{\prime}\right|=|B|-\left|A^{\prime} \cap B\right| \leq(k+2)-(k+1)=1$. As observed earlier, $N(u) \subseteq A \cup B$. Since $A \cap A^{\prime}=\emptyset$ and $u \in A$, we note that $u \notin A^{\prime}$, and since it is not either in $B^{\prime}$, $N(u) \subseteq A \cup\left(B \backslash A^{\prime}\right)$. Hence, $k+2=d(u)=d_{A}(u)+d_{B \backslash A^{\prime}}(u) \leq|A \backslash\{u\}|+\left|B \backslash A^{\prime}\right| \leq$ $(k+1)+1=k+2$. Therefore, we must have equality throughout this inequality chain, implying that $|A|=k+2,\left|B \backslash A^{\prime}\right|=1, d_{A}(u)=k+1$, and $d_{B \backslash A^{\prime}}(u)=1$. In particular, we note that the vertex $u$ is adjacent to every vertex in $A \backslash\{u\}$. Let $B \backslash A^{\prime}=\{w\}$. Then, $u w \in E$. Since $w \in B$, by property (P3), $d_{A}(w)=k+1$. Let $z$ be a neighbor of $w$ in $A \backslash\{u\}=A \cap B^{\prime}$. As observed earlier, [ $\left.A^{\prime} \cap B, A \cap B^{\prime}\right]$ is full and $\left|A^{\prime} \cap B\right|=k+1$. Hence, $z$ is adjacent to $k+1$ vertices in $A^{\prime} \cap B$ and is adjacent to both $u \in A \backslash A^{\prime}$ and $w \in B \backslash A^{\prime}$, implying that $d(z) \geq k+3$, a contradiction. Therefore, $A \backslash B^{\prime}=\emptyset$, and so $A \subseteq B^{\prime}$. Analogously, $A^{\prime} \subseteq B$.

By property (P4), the set $B^{\prime}$ is an independent set. Hence, since $A \subseteq B^{\prime}$, the set $A$ is an independent set, implying by property (P2) that $N(v) \subseteq B$ for every vertex $v \in A$. By property (P7), $|B| \leq k+2$. Hence, $k+2=d(v) \leq|B| \leq k+2$. Therefore, we must have equality throughout this inequality chain, implying that $|B|=k+2$ 
and $N(v)=B$ for every $v \in A$. Hence, $[A, B]$ is full. Analogously, $\left|B^{\prime}\right|=k+2$ and $\left[A^{\prime}, B^{\prime}\right]$ is full. If $y \in B \cap B^{\prime}$, then since $A \cap A^{\prime}=\emptyset$, we have by property (P3) that $k+2=d(y) \geq d_{A}(y)+d_{A^{\prime}}(y)=2(k+1)$, and so $k \leq 0$, a contradiction. Hence, $B \cap B^{\prime}=\emptyset$. Thus since $A \subseteq B^{\prime}$ and $A^{\prime} \subseteq B^{\prime}$, and $|B|=\left|B^{\prime}\right|=k+2$, the graph $K_{k+2, k+2}-e$ is a spanning subgraph of $G\left[\bar{A} \cup A^{\prime} \cup B \cup B^{\prime}\right]$.

5. Proof of Theorem 2.1. In this section, we present a proof of our main result, namely, Theorem 2.1. For this purpose, we first prove four preliminary lemmas. We define the reduced graph of $G$, denoted $G^{*}$, to be the graph obtained from $G$ by deleting all edges that do not belong to a cycle of length 3 or 4 in $G$.

LEMMA 5.1. For $k \geq 1$, let $G$ be a connected $(k+2)$-regular graph of order at least $2 k+4$. Suppose $G$ contains a subgraph $F_{k}$ of type- $\mathcal{F}_{k}$, and let $\left(X_{1}, X_{2},\{u, x, y\}\right)$ be the partition of $V\left(F_{k}\right)$, as defined in Definition 3.1. Then the subgraph $F_{k}$ is a component of $G^{*}$ (possibly with the edge $x y$ ).

Proof. Suppose that $G=(V, E)$ contains a subgraph $F_{k}$ of type- $\mathcal{F}_{k}$. By definition, we note that $k \geq 2$. We show first that every edge of $F_{k}$ is an edge of the reduced graph $G^{*}$. Let $\left(X_{1}, X_{2},\{u, x, y\}\right)$ be the partition of $V\left(F_{k}\right)$, as defined in Definition 3.1.

Let $e$ be an arbitrary edge in $\left[X_{1}, X_{2}\right]$. Let $e=v w$, where $v \in X_{2}$ (and $w \in X_{1}$ ). Since $d_{X_{1} \cup\{u\}}(v)=k+1$, the vertex $v$ is adjacent to all but one of the $k+2$ vertices in the set $X_{1} \cup\{u\}$. In particular, the vertex $v$ is adjacent to at least $k \geq 2$ vertices in $X_{1}$. Let $w^{\prime}$ be a neighbor of $v$ in $X_{1}$ different from $w$. Then, $v w y w^{\prime} v$ is a 4-cycle in $G$ containing the edge $e$. Hence, every edge in $\left[X_{1}, X_{2}\right]$ is contained in a 4-cycle in $G$.

Let $f$ be an arbitrary edge in $\left[X_{1},\{y\}\right]$. Let $f^{\prime}$ be an edge in $\left[X_{1},\{y\}\right]$ different from $f$, and let $f=w y$ and $f^{\prime}=w^{\prime} y$. If $w$ and $w^{\prime}$ have no common neighbor in $X_{2} \cup\{u\}$, then $k+2=\left|X_{2} \cup\{u\}\right| \geq d_{X_{2} \cup\{u\}}(w)+d_{X_{2} \cup\{u\}}\left(w^{\prime}\right)=2(k+1)$, and so $k \leq 0$, a contradiction. Hence, $w$ and $w^{\prime}$ have a common neighbor in $X_{2} \cup\{u\}$, say, $z$. But then $y w z w^{\prime} y$ is a 4-cycle in $G$ containing the edge $f$. Hence, every edge in $\left[X_{1},\{y\}\right]$ is contained in a 4-cycle in $G$. Analogously, every edge in $\left[X_{2},\{x\}\right]$ is contained in a 4 -cycle in $G$.

It remains to show that every edge incident with $u$ belongs to a 3-cycle or a 4-cycle. Let $g$ be an arbitrary edge incident with $u$. Let $g=u v$, where we may assume that $v \in X_{2}$. If $d_{X_{2}}(u) \geq 2$, then let $v^{\prime}$ be a neighbor of $u$ in $X_{2}$ different from $v$. Then, $u v x v^{\prime} u$ is a 4-cycle in $G$ containing the edge $g$. If $d_{X_{2}}(u)=1$, then $d_{X_{1}}(u)=\left|X_{1}\right|=k+1$. In this case, given any neighbor $u_{1}$ of $u$ in $X_{1}, u u_{1} v u$ is a 3 -cycle in $G$ containing the edge $g$. Hence, every edge incident with $u$ belongs to a 3 -cycle or a 4-cycle.

We have therefore shown that every edge in $F_{k}$ belongs to a 3 -cycle or a 4-cycle and therefore belongs to the reduced graph $G^{*}$. We prove next that $F_{k}$ is a component of $G^{*}$. If $x y \in E$, then the graph $G$ is determined and $G=F_{k}+x y$, the vertices of $F_{k}$ indeed forming a component of $G^{*}$. Otherwise, by the $(k+2)$-regularity of $G$, there is exactly one edge incident with each of $x$ and $y$ that does not belong to $F_{k}$. Let $e_{x}$ and $e_{y}$ be the edges incident with $x$ and $y$, respectively, that do not belong to $F_{k}$. By assumption, $e_{x} \neq e_{y}$. Every cycle containing the edge $e_{x}$ contains a path in $F_{k}$ from $x$ to $y$ as well as the edge $e_{y}$. Since the distance between $x$ and $y$ in $F_{k}$ is 3 , such a cycle has length at least 5 , implying that the edge $e_{x}$ does not belong to $G^{*}$. Analogously, the edge $e_{y}$ does not belong to $G^{*}$. Therefore, $F_{k}$ is a component of $G^{*}$.

Lemma 5.2. For $k \geq 1$, let $G$ be a connected $(k+2)$-regular graph of order at least $2 k+4$. If $G \neq K_{k+2, k+2}$ and $G$ contains a subgraph isomorphic to $K_{k+2, k+2}-e$, then such a subgraph is a component of $G^{*}$. 
Proof. Suppose that $G \neq K_{k+2, k+2}$ but $G$ contains a subgraph, $F$, say, isomorphic to $K_{k+2, k+2}-e$. Since every edge of $F$ belongs to a 4-cycle, every edge of $F$ is an edge of the reduced graph $G^{*}$. It remains to prove that $F$ is a component of $G^{*}$. Let $x$ and $y$ be the ends of the nonedge $e$ in $F$. By the $(k+2)$-regularity of $G$, there is exactly one edge incident that does not belong to $F$. Let $e_{x}$ and $e_{y}$ be the edges incident with $x$ and $y$, respectively, that do not belong to $F$. Since $G \neq K_{k+2, k+2}$, we note that $e_{x} \neq e_{y}$. Every cycle containing the edge $e_{x}$ contains a path in $F$ from $x$ to $y$ as well as the edge $e_{y}$. Since the distance between $x$ and $y$ in $F$ is 3 , such a cycle has length at least 5 , implying that the edge $e_{x}$ does not belong to $G^{*}$. Analogously, the edge $e_{y}$ does not belong to $G^{*}$. Therefore, $F$ is a component of $G^{*}$.

Recall that if $X$ and $Y$ are subsets of vertices and there is no edge in $[X, Y]$, then we say that $[X, Y]$ is empty.

Lemma 5.3. For $k \geq 1$, let $G=(V, E)$ be a connected $(k+2)$-regular graph of order at least $2 k+4$. Suppose there exist subsets $A, B$, and $S$ of vertices in $G$ such that $M=\mathcal{P}^{\infty}(S), \bar{M}=V \backslash M$, and $B=N(A) \cap M$, and such that the following holds:

(a) $A \cap M=\emptyset$.

(b) $|A| \leq k+2$.

(c) $[A \cup B, \bar{M} \backslash A]$ is empty.

Then, the sets $A$ and $B$ induce an $(A, B)$-configuration in $G$.

Proof. In order to prove that $A$ and $B$ induce an $(A, B)$-configuration in $G$, we need to prove that properties (P1) to (P4) hold.

By part (c) in the statement of the lemma, $N(A) \subseteq A \cup M$. Therefore, $N(A) \backslash A=$ $N(A) \cap M=B$. This proves property (P2).

By part (c) in the statement of the lemma, $N(B) \subseteq A \cup M$. Therefore, $N(B) \cap$ $\bar{M}=A$. Let $x \in B$. Then, $x \in N(A)$, and so $d_{A}(x) \geq 1$. By definition, $M=\mathcal{P}^{\infty}(S)$. Hence, every vertex in $M$ that has a neighbor in $\bar{M}$ does not belong to the set $S$ and contains at least $k+1$ neighbors in $\bar{M}$. In particular, $x \notin S$ and $d_{A}(x) \geq k+1$. Since $x \in M \backslash S$, there exists a vertex $v \in M$ adjacent to $x$, and so $d_{M}(x) \geq 1$. Hence, $k+2=d(x) \geq d_{M}(x)+d_{A}(x) \geq 1+(k+1)=k+2$. Therefore, we must have equality throughout this inequality chain. In particular, $d_{A}(x)=k+1$. Since $x$ is an arbitrary vertex in $B$, this proves property (P3).

As an immediate consequence of property (P3), we have that $|A| \geq k+1$, which together with part (b) in the statement of the lemma proves property (P1).

It remains to prove that $B$ is an independent set. Suppose that $u, v \in B$ with $u v \in E$. By property (P3), $d_{A}(u)=d_{A}(v)=k+1$, and so by the $(k+2)$-regularity of $G$ we have that $N(u) \subseteq A \cup\{v\}$ and $N(v) \subseteq A \cup\{u\}$. Thus, $u$ is the only neighbor of $v$ in $M$ and $v$ is the only neighbor of $u$ in $M$. If $S \cap\{u, v\}=\emptyset$, then by part (a) in the statement of the lemma, neither $u$ nor $v$ belong to the set $M$, a contradiction. Hence, $|S \cap\{u, v\}| \geq 1$. Renaming $u$ and $v$ if necessary, we may assume that $u \in S$. But then $N_{A}(u) \subset M$, contradicting part (a). Therefore, $B$ is an independent set. This proves property $(\mathrm{P} 4)$.

We are now in a position to prove our final preliminary lemma. Recall that if $F_{k}$ is a graph in the family $\mathcal{F}_{k}$ with vertex partition as defined in Definition 3.1, then we call the vertex $u$ the focal vertex of $F_{k}$.

Lemma 5.4. For $k \geq 1$, let $G=(V, E)$ be a connected $(k+2)$-regular graph of order at least $2 k+4$ such that $G \neq K_{k+2, k+2}$ and $\gamma_{\mathrm{P}, k}(G)>1$. Then there exists a sequence $S_{0}, \ldots, S_{\ell}$ such that the following holds:

(a) $S_{0}$ is a packing in $G$.

(b) For all $i \geq 0,\left|S_{i+1}\right|=\left|S_{i}\right|+1$. 
(c) $\left|\mathcal{P}^{\infty}\left(S_{i+1}\right)\right| \geq\left|\mathcal{P}^{\infty}\left(S_{i}\right)\right|+k+3$.

(d) $\mathcal{P}^{\infty}\left(S_{\ell}\right)=V$.

Proof. We first construct a packing $S_{0}$ in $G$ as follows. Let $P_{0}$ be the set consisting of the focal vertex from each component of type- $\mathcal{F}_{k}$ in the reduced graph $G^{*}$ and a vertex of degree $k+2$ from every component of $G^{*}$ isomorphic to $K_{k+2, k+2}-e$. It follows from Lemma 5.1 and Lemma 5.2 that the set $P_{0}$ so constructed is a packing in the graph $G$.

Let $A$ and $B$ be subsets of vertices in $G$ that induce an $(A, B)$-configuration that is not totally included in a component of type- $\mathcal{F}_{k}$ or in a component isomorphic to $K_{k+2, k+2}-e$ in $G^{*}$. Let $A^{\prime}$ and $B^{\prime}$ be subsets of vertices in $G$ that induce an $\left(A^{\prime}, B^{\prime}\right)$-configuration with $A \neq A^{\prime}$. By Lemma 4.3(c), if $\left|A \cap A^{\prime}\right| \geq 1$, then $\left|A \cap A^{\prime}\right| \in\{1, k, k+1\}$. By assumption, $\gamma_{\mathrm{P}, k}(G)>1$, and so by Lemma 4.5 we note that $\left|A \cap A^{\prime}\right| \neq k+1$. Further, by assumption, $G \neq K_{k+2, k+2}$, and so by Lemma 4.4 we note that $\left|A \cap A^{\prime}\right| \neq k$ or $k=1$. If $\left|A \cap A^{\prime}\right|=1$, then by Lemma 4.6 either the $(A, B)$-configuration is included in a component of type- $\mathcal{F}_{k}$ in $G^{*}$, or $k=1$ and $G=K_{3,3}$, contradicting in both cases the assumptions. It follows that $A \cap A^{\prime}=\emptyset$. This in turn implies by Lemma 4.7 that $B \cap B^{\prime}=\emptyset$. Observe now that $A \cap B^{\prime}=\emptyset$. Otherwise the $(A, B)$-configuration is contained in a component isomorphic to $K_{k+2, k+2}-e$ in $G^{*}$ by Lemma 4.8, contradicting the choice of $A$ and $B$. Similarly we have $B \cap A^{\prime}=\emptyset$. It follows that the $(A, B)$-configuration and the $\left(A^{\prime}, B^{\prime}\right)$-configuration do not intersect. Also, since every vertex of a subgraph of type- $\mathcal{F}_{k}$ or a subgraph isomorphic to $K_{k+2, k+2}-e$ is contained in some $\left(A^{\prime}, B^{\prime}\right)$-configuration, the $(A, B)$-configuration is totally disjoint from any such subgraph. From each such $(A, B)$-configuration, we select an arbitrary vertex from $A$ and add it to the set $P_{0}$. Let $P_{1}$ denote the resulting set of vertices. Since these $(A, B)$-configurations (that do not belong to a component of type- $\mathcal{F}_{k}$ in $G^{*}$ or to a component of $G^{*}$ isomorphic to $K_{k+2, k+2}-e$ ) do not intersect, the set $P_{1}$ is a packing in $G$.

By construction, if $A$ and $B$ are subsets of vertices in $G$ that induce an $(A, B)$ configuration, then $A$ and $N\left[P_{1}\right]$ intersect. Finally, we extend the packing $P_{1}$ into a maximal packing of $G$ and we let $S_{0}$ denote the resulting maximal packing. This establishes the packing in the statement of part (a).

We now prove part (b) and part (c) by induction on $i \geq 0$. If $\mathcal{P}^{\infty}\left(S_{0}\right)=V$, then there is nothing to prove. Hence, we may assume that $\mathcal{P}^{\infty}\left(S_{0}\right) \neq V$. Let $i \geq 0$ and suppose that $S_{i}$ exists and $\mathcal{P}^{\infty}\left(S_{i}\right) \neq V$. Let $u \in \mathcal{P}^{\infty}\left(S_{i}\right)$ such that $N(u) \backslash \mathcal{P}^{\infty}\left(S_{i}\right) \neq \emptyset$. Let $A=N(u) \backslash \mathcal{P}^{\infty}\left(S_{i}\right)$ and denote by $M=\mathcal{P}^{\infty}\left(S_{i}\right)$ and $\bar{M}=V \backslash \mathcal{P}^{\infty}\left(S_{i}\right)$. Since $N[u] \not \subset \mathcal{P}^{\infty}\left(S_{i}\right)$, we note that $u \notin S_{i}, d_{M}(u) \geq 1$ and $d_{A}(u) \geq k+1$. Therefore, by the $(k+2)$-regularity of $G$, we note that $d_{M}(u)=1$ and $d_{A}(u)=k+1$, and so $|A|=k+1$.

Suppose that there exists a vertex $x \in A$ such that $d_{\bar{M} \backslash A}(x) \geq 2$. In this case, we define $S_{i+1}=S_{i} \cup\{x\}$ and we let $j$ be the minimum integer such that $\mathcal{P}^{j}\left(S_{i}\right)=$ $\mathcal{P}^{\infty}\left(S_{i}\right)$. Then, $N[u] \backslash \mathcal{P}^{j}\left(S_{i+1}\right) \subseteq\left(N[u] \backslash \mathcal{P}^{j}\left(S_{i}\right)\right) \backslash\{x\}$, and so $N[u] \backslash \mathcal{P}^{j}\left(S_{i+1}\right) \leq k$, implying that $N[u] \subseteq \mathcal{P}^{j+1}\left(S_{i+1}\right)$. Also, $N[x] \subseteq \mathcal{P}^{0}\left(S_{i+1}\right) \subseteq \mathcal{P}^{j+1}\left(S_{i+1}\right)$. Therefore, $\left|\mathcal{P}^{\infty}\left(S_{i+1}\right)\right| \geq\left|\mathcal{P}^{\infty}\left(S_{i}\right)\right|+k+3$. Hence we may assume that $d_{\bar{M} \backslash A}(x) \leq 1$ for every vertex $x \in A$, for otherwise part (b) and part (c) follow as desired.

Let $B=N(A) \cap M$. From the choice of our packing $S_{0}$ and since $A \subseteq \bar{M}$, the sets $A$ and $B$ do not induce an $(A, B)$-configuration in $G$. We deduce, therefore, by Lemma 5.3 that $[A \cup B, \bar{M} \backslash A]$ is not empty. Thus there exists an edge $v w \in E$ where $v \in A \cup B$ and $w \in \bar{M} \backslash A$. Let $A^{\prime}=A \cup\{w\}$ and let $B^{\prime}=N_{M}\left(A^{\prime}\right)$. Once again by our construction of the packing $S_{0}$, the sets $A^{\prime}$ and $B^{\prime}$ do not induce an 
$\left(A^{\prime}, B^{\prime}\right)$-configuration in $G$. Hence by Lemma 5.3 there exists an edge $v^{\prime} w^{\prime} \in E$ where $v^{\prime} \in A^{\prime} \cup B^{\prime}$ and $w^{\prime} \in \bar{M} \backslash A^{\prime}$.

We now define $S_{i+1}=S_{i} \cup\{w\}$. We show that $\mathcal{P}^{\infty}\left(S_{i+1}\right)$ contains $A^{\prime} \cup\left\{w^{\prime}\right\}$. Since $w \in S_{i+1}$, we note that $N[w] \subseteq \mathcal{P}^{0}\left(S_{i+1}\right)$, and so $\{v, w\} \subset \mathcal{P}^{0}\left(S_{i+1}\right)$. Let $j$ be the smallest integer such that $\mathcal{P}^{\infty}\left(S_{i}\right)=\mathcal{P}^{j}\left(S_{i}\right)$.

On the one hand, suppose that $v \in B$. Since $B=N_{M}(A)$, we note that $d_{A}(v) \geq 1$ and we let $x \in N_{A}(v)$. Since $v \in M$ and $w \in S_{i+1}$, we have that

$$
N[v] \backslash \mathcal{P}^{j}\left(S_{i+1}\right) \subseteq N[v] \backslash\left(\mathcal{P}^{j}\left(S_{i}\right) \cup\{w\}\right),
$$

and so

$$
\left|N[v] \backslash \mathcal{P}^{j}\left(S_{i+1}\right)\right| \leq\left|N[v] \backslash \mathcal{P}^{j}\left(S_{i}\right)\right|-1=(k+1)-1=k,
$$

implying that $N[v] \subseteq \mathcal{P}^{j+1}\left(S_{i+1}\right)$ and therefore that $x \in \mathcal{P}^{j+1}\left(S_{i+1}\right)$ (where we recall that $x \in A$ ). On the other hand, suppose that $v \notin B$. Then, $v \in A$. Further, $v \in N[u] \cap N[w]$. Since $w \in S_{i+1}$, we note that $v \in \mathcal{P}^{0}\left(S_{i+1}\right) \subseteq \mathcal{P}^{j}\left(S_{i+1}\right)$. Hence, in both cases there is a vertex in the set $A$ that belongs to the set $\mathcal{P}^{j+1}\left(S_{i+1}\right)$, implying that

$$
\left|N[u] \backslash \mathcal{P}^{j+1}\left(S_{i+1}\right)\right| \leq\left|N[u] \backslash \mathcal{P}^{j}\left(S_{i}\right)\right|-1=|A|-1=k .
$$

Hence, $A \subseteq N[u] \subseteq \mathcal{P}^{j+2}\left(S_{i+1}\right)$. It remains to show that $w^{\prime} \in \mathcal{P}^{\infty}\left(S_{i+1}\right)$. If $v^{\prime}=w$, then $w^{\prime} \in \mathcal{P}^{0}\left(S_{i+1}\right)$, and we are done. If $v^{\prime} \in A$, then $v^{\prime} \in \mathcal{P}^{j+2}\left(S_{i+1}\right)$, and since $d_{\bar{M} \backslash A}\left(v^{\prime}\right) \leq 1$, the vertex $w^{\prime}$ is the only neighbor of $v^{\prime}$ not in $\mathcal{P}^{j+2}\left(S_{i+1}\right)$, implying that $w^{\prime} \in \mathcal{P}^{j+3}\left(S_{i+1}\right)$. Finally, if $v^{\prime} \in B^{\prime}$, then $N\left(v^{\prime}\right) \cap A^{\prime} \neq \emptyset$, and so

$$
\left|N\left(v^{\prime}\right) \backslash \mathcal{P}^{j+2}\left(S_{i+1}\right)\right| \leq\left|N\left(v^{\prime}\right) \backslash M\right|-\left|N\left(v^{\prime}\right) \cap A^{\prime}\right| \leq(k+1)-1=k,
$$

implying that $N\left(v^{\prime}\right) \subseteq \mathcal{P}^{j+3}\left(S_{i+1}\right)$ and therefore that $w^{\prime} \in \mathcal{P}^{j+3}\left(S_{i+1}\right)$. In all cases, we have shown that $w^{\prime} \in \mathcal{P}^{\infty}\left(S_{i+1}\right)$. Hence, $A \cup\left\{w, w^{\prime}\right\} \subseteq \mathcal{P}^{\infty}\left(S_{i+1}\right) \backslash \mathcal{P}^{\infty}\left(S_{i}\right)$, and so $\left|\mathcal{P}^{\infty}\left(S_{i+1}\right)\right| \geq\left|\mathcal{P}^{\infty}\left(S_{i}\right)\right|+k+3$. This establishes part (b) and part (c).

We have shown that for $i \geq 0$, if the set $S_{i}$ exists and $\mathcal{P}^{\infty}\left(S_{i}\right) \neq V$, then $\left|\mathcal{P}^{\infty}\left(S_{i+1}\right)\right|>\left|\mathcal{P}^{\infty}\left(S_{i}\right)\right|$. Hence, since $|V|$ is finite, there must exist an integer $\ell$ such that $\mathcal{P}^{\infty}\left(S_{\ell}\right)=V$, which establishes part (d).

We are now in a position to prove our main result, namely, Theorem 2.1. Recall its statement.

THEOREM 2.1. Let $k \geq 1$ and let $G$ be a connected $(k+2)$-regular graph of order $n$. If $G \neq K_{k+2, k+2}$, then $\gamma_{\mathrm{P}, k}(G) \leq n /(k+3)$, and this bound is tight.

Proof of Theorem 2.1. If $\gamma_{\mathrm{P}, k}(G)=1$, the result is immediate since $n \geq k+3$. Hence, we may assume that $\gamma_{\mathrm{P}, k}(G) \geq 2$. In particular this implies that $n \geq 2 k+4$, for otherwise any vertex in $G$ is a $\mathrm{kPD}$-set in $G$ (and $\gamma_{\mathrm{P}, k}(G)=1$ ). We may therefore apply Lemma 5.4 to the graph $G$. Let $S_{0}, \ldots, S_{\ell}$ be a sequence satisfying properties (a)-(d) in the statement of Lemma 5.4 with $\ell$ as small as possible. By Lemma 5.4(d), the set $S_{\ell}$ is a kPD-set in $G$, and so $\gamma_{\mathrm{P}, k}(G) \leq\left|S_{\ell}\right|$. Since $S_{0}$ is a packing in $G$, we have that $\left|\mathcal{P}^{0}\left(S_{0}\right)\right|=\left|N\left[S_{0}\right]\right|=\left|S_{0}\right| \times(k+3)$. If $\ell=0$, then $n=(k+3)\left|S_{0}\right|$ and $\gamma_{\mathrm{P}, k}(G) \leq\left|S_{0}\right|=n /(k+3)$, and we are done. Hence, we may assume that $\ell \geq 1$. By Lemma 5.4(b), $\left|S_{\ell}\right|=\left|S_{0}\right|+\ell$. By our choice of $\ell$, we have by Lemma 5.4(c) that $\left|\mathcal{P}^{\infty}\left(S_{i+1}\right)\right| \geq\left|\mathcal{P}^{\infty}\left(S_{i}\right)\right|+k+3$ for $0 \leq i \leq \ell-1$, and so

$$
n=\left|\mathcal{P}^{\infty}\left(S_{\ell}\right)\right| \geq\left|\mathcal{P}^{0}\left(S_{0}\right)\right|+\ell(k+3)=\left(\left|S_{0}\right|+\ell\right)(k+3)=\left|S_{\ell}\right|(k+3),
$$

and so $\gamma_{\mathrm{P}, k}(G) \leq\left|S_{\ell}\right| \leq n /(k+3)$, as desired. This proves the desired upper bound. 


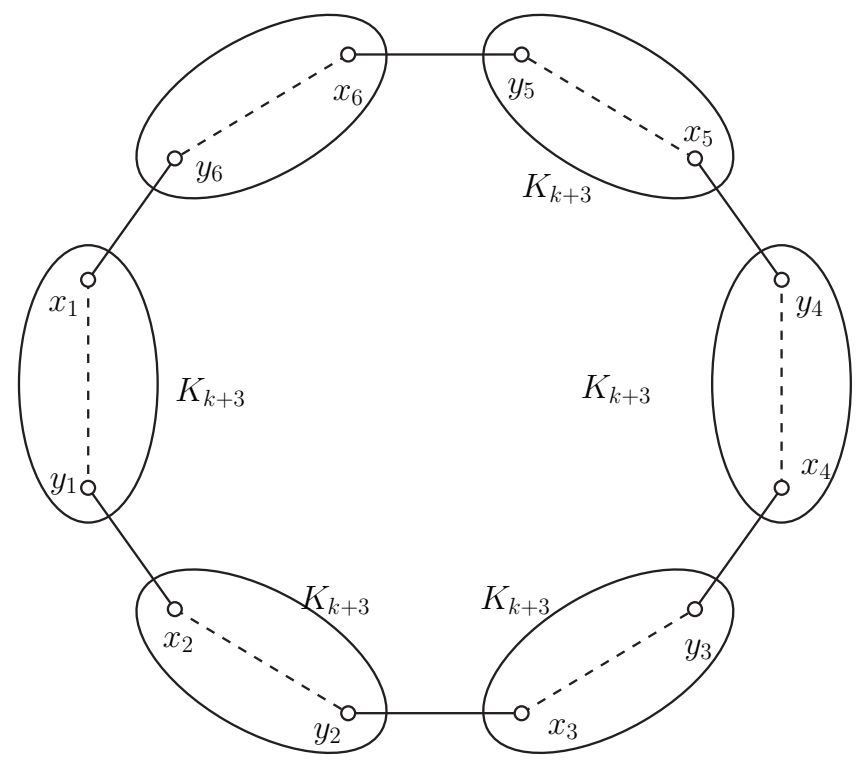

FIG. 5.1. The graph $G_{k, 6}$.

That this bound is tight, may be seen as follows. Let $k \geq 1$ and $r \geq 2$ be integers. For $1 \leq i \leq r$, let $G_{i}$ be a complete graph on $k+3$ vertices minus one edge $x_{i} y_{i}$, and so $G_{i} \cong K_{k+3}-e$. Let $G_{k, r}$ be the graph obtained from the disjoint union of the graphs $G_{1}, G_{2}, \ldots, G_{r}$ by adding the edges $y_{i} x_{i+1}$ for $i=1,2, \ldots, r$, where $x_{r+1}=x_{1}$ (see Figure 5.1). Then, $G_{k, r}$ is a connected (k+2)-regular graph of order $n=r(k+3)$. Let $S$ be an arbitrary kPD-set in $G_{k, r}$. If $S \cap V\left(G_{i}\right)=\emptyset$, then no vertex in $V\left(G_{i}\right) \backslash\left\{x_{i}, y_{i}\right\}$ belongs to the set $\mathcal{P}^{\infty}(S)$, contradicting the assumption that $S$ is a kPD-set in $G_{k, r}$. Hence, $\left|S \cap V\left(G_{i}\right)\right| \geq 1$ for all $i, 1 \leq i \leq r$, implying that $\gamma_{\mathrm{P}, k}\left(G_{k, r}\right) \geq r=n /(k+3)$. However, as shown earlier, if $G$ is a connected $(k+2)$-regular graph of order $n$ and $G \neq K_{k+2, k+2}$, then $\gamma_{\mathrm{P}, k}(G) \leq n /(k+3)$. Consequently, $\gamma_{\mathrm{P}, k}\left(G_{k, r}\right)=n /(k+3)$. This completes the proof of Theorem 2.1.

6. Conclusion. We pose the following conjecture that we have yet to settle.

ConjeCture 1 . For $k \geq 1$ and $r \geq 3$, if $G \neq K_{r, r}$ is a connected $r$-regular graph of order $n$, then $\gamma_{\mathrm{P}, k}(G) \leq n /(r+1)$.

We remark that if $k \geq r-1$ in the statement of Conjecture 1 , then $\gamma_{\mathrm{P}, k}(G)=$ $1 \leq n /(r+1)$ and the conjecture is trivially true. If $k=r-2$, then the conjecture is true by our main result, namely, Theorem 2.1 . When $k \leq r-3$, we have yet to settle the conjecture. We close with the following question.

QUESTION 1. For $r \geq 3$, let $G \neq K_{r, r}$ be a connected $r$-regular graph of order $n$. Determine the smallest positive value, $k_{\min }(r)$, of $k$ such that $\gamma_{\mathrm{P}, k}(G) \leq n /(r+1)$.

We remark that by our main result, namely Theorem 2.1 , we know that $k_{\min }(r) \leq$ $r-2$. If Conjecture 1 is true, then $k_{\min }(r)=1$.

\section{REFERENCES}

[1] A. Aazami, Domination in graphs with bounded propagation: Algorithms, formulations and hardness results, J. Comb. Optim., 19 (2010), pp. 429-456.

[2] A. Aazami And M. D. Stilp, Approximation algorithms and hardness for domination with propagation, SIAM J. Discrete Math., 23 (2009), pp. 1382-1399. 
[3] T. L. Baldwin, L. Mili, M. B. Boisen JR., And R. Adapa, Power system observability with minimal phasor measurement placement, IEEE Trans. Power Systems, 8 (1993), pp. 707715.

[4] G. J. Chang, P. Dorbec, M. Montassier, and A. Raspaud, Generalized power domination of graphs, Discrete Appl. Math., 160 (2012), pp. 1691-1698.

[5] P. Dorbec, M. Mollard, S. Klavžar, and S. Špacapan, Power domination in product graphs, SIAM J. Discrete Math., 22 (2008), pp. 554-567.

[6] M. Dorfling and M. A. Henning, A note on power domination in grid graphs, Discrete Appl. Math., 154 (2006), pp. 1023-1027.

[7] J. Guo, R. Niedermeier, AND D. RAible, Improved algorithms and complexity results for power domination in graphs, Lecture Notes in Comput. Sci. 3623, Springer, Berlin, 2005, pp. $172-184$.

[8] J. Guo, R. Niedermeier, And D. Raible, Improved algorithms and complexity results for power domination in graphs, Algorithmica, 52 (2008), pp. 177-202.

[9] T. W. Haynes, S. M. Hedetniemi, S. T. Hedetniemi, and M. A. Henning, Domination in graphs applied to electric power networks, SIAM J. Discrete Math., 15 (2002), pp. 519-529.

[10] T. W. Haynes, S. T. Hedetniemi, and P. J. Slater, Fundamentals of Domination in Graphs, Marcel Dekker, New York, 1998.

[11] J. Kneis, D. Mölle, S. Richter, and P. Rossmanith, Parameterized power domination complexity, Inform. Process. Lett. 98 (2006), pp. 145-149.

[12] C.-S. LiaO And D.-T. Lee, Power domination problem in graphs, Lecture Notes in Comput. Sci. 3595, 2005, Springer, Berlin, pp. 818-828.

[13] L. Mili, T. Baldwin, And A. Phadke, Phasor measurement placement for voltage and stability monitoring and control, in Proceedings of the EPRI-NSF Workshop on Application of Advanced Mathematics to Power Systems, San Francisco, CA, 1991.

[14] G. Xu, L. Kang, E. Shan, And M. Zhao, Power domination in block graphs, Theoret. Comput. Sci., 359 (2006), pp. 299-305.

[15] M. ZhaO And L. KAng, Power domination in planar graphs with small diameter, J. Shanghai Univ., 11 (2007), pp. 218-222.

[16] M. Zhao, L. Kang, and G. J. Chang, Power domination in graphs, Discrete Math., 306 (2006), pp. 1812-1816. 